\title{
¿Son los niveles de prolactina y estradiol al tercer mes posparto factores predictores del tiempo de aparición de la primera menstruación posparto?: Modelo predictivo mediante análisis de sobrevida
}

\author{
Patricio Valdés G ${ }^{1,2}$, Juan J 0 rellana $\mathbf{C}^{2,3 a}$. \\ Post suckling prolactin and estradiol \\ levels as predictors of the time \\ of appearance of the first post partum \\ menstruation
}

Background: The variability in the duration of lactational amenorrhoea (LA) lead to develop statistical multivariate models to predict the risk of the appearance of the first postpartum menstruation. Aim: To estimate the probability of recovering the first postpartum menstruation by means of a survival analysis, including hormonal levels and other parameters as predictor variables. Material and Methods: Eighty one mothers in exclusive breastfeeding until the sixth postpartum month, in whom estradiol, basal and post suckling prolactin were measured at the third post partum month, were studied. The variables that better predict the appearance of the first menstruation between the $3^{\text {rd }}$ and $12^{\text {th }}$ postpartum months, were identified using a Cox model survival analysis. Results: The median amenorrhea survival time (the lapse when the chance of recovering menstruation is $50 \%$ ) was 209 days from delivery. Dichotomized estradiol and post suckling prolactin were the only significant variables that predicted the return of menstruation, with cutoff points of $190 \mathrm{pmol} /$ and 2,550 mIU/L, respectively. Conclusions: Post suckling prolantin and estradiol levels, measured at the third post partum month, are predictors for the time of appearance of the first postpartum menstruation (Rev Méd Chile 2007; 135: 419-26).

(Key words: Amenorrhea; Estradiol; Lactation; Prolactin)

Recibido el 5 de septiembre, 2005. Aceptado el 29 de septiembre, 2006.

${ }^{1}$ Departamento de Obstetricia y Ginecología. ${ }^{2}$ Centro de Capacitación Investigación y Gestión para la Salud Basada en Evidencia (CIGES). ${ }^{3}$ Departamento de Salud Pública, Facultad de Medicina, Universidad de La Frontera.

aProfesor de Estado en Matemáticas

Correspondencia a: Patricio Valdés G. Departamento de

Obstetricia y Ginecología, Facultad de Medicina, Universi-

dad de La Frontera. M. Montt 112, Temuco. Fax: 45-325745.

E mail: pvaldes@ufro.cl 
T a variabilidad en la duración de la amenorrea Lpor lactancia (AL) entre las mujeres de una misma o diferentes poblaciones ${ }^{1-4}$, ha llevado a buscar elementos clínicos y de laboratorio que se puedan asociar a la aparición de la primera menstruación postparto durante el periodo de lactancia exclusiva. En diseños de cohortes realizados en mujeres urbanas de Chile, se ha encontrado una asociación entre la duración de AL y los niveles plasmáticos de estradiol (E), prolactina b (PRLb) (antes de un episodio de succión) y prolactina $\mathrm{p}$ (PRLp) (después de un episodio de succión) al tercer mes postparto ${ }^{5-7}$. Estos diseños establecen una evaluación dicotómica de la AL (AL larga/AL corta) de acuerdo al punto de corte de 6 meses postparto. Los casos que permanecen en amenorrea más allá de los seis meses postparto, demuestran concentraciones plasmáticas más altas de PRLb y niveles más bajos de $\mathrm{E}$ al tercer mes postparto, respecto a las que presentaron su primera menstruación antes de los seis meses $^{6,7}$. Además, en otros trabajos, se establece que la razón PRLp/E se puede proponer como un posible factor predictivo de la duración de la $\mathrm{AL}^{8,9}$. Estos estudios avalan la búsqueda de modelos estadísticos multivariados de aceptable validez y precisión para determinar el riesgo de aparición de la primera menstruación en el periodo del amamantamiento.

El presente trabajo estudia una cohorte de madres urbanas chilenas en lactancia exclusiva hasta el sexto mes postparto y mediante un modelo de análisis de sobrevida, se estima la probabilidad de permanecer amenorreica más allá de un tiempo definido, tomando como variables predictoras la PRLb, PRLp, E, la razón PRLp/E al tercer mes postparto, edad, paridad, etnia e índice de masa corporal (IMC) materno.

Se propone un modelo de análisis multivariado que permita establecer con mayor precisión el tiempo de duración de AL.

\section{MATERIAL Y MÉTODO}

Se realiza un análisis de sobrevida de la aparición de la primera menstruación en una serie de 81 madres urbanas de la ciudad de Temuco, que estuvieron en lactancia exclusiva hasta el sexto mes postparto y cuyo seguimiento se extendió desde el primer día hasta los 12 meses postparto. En estas madres se realizó una medición de las concentraciones plasmáticas de PRLb, PRLp y E al tercer mes postparto. Previa a la toma de muestra para al estudio, todas las madres firmaron un consentimiento informado el cual fue autorizado por el Comité Ético-Científico de la Facultad de Medicina de la Universidad de La Frontera.

En el momento del examen, se registraron los datos de las variables biodemográficas (edad, etnia, paridad e IMC).

Estas informaciones permiten:

1. Identificar las variables que mejor predicen la aparición de la primera menstruación postparto a un tiempo determinado entre el tercery décimo segundo mes postparto. 2. Identificar los puntos de corte de las variables continuas (niveles hormonales e IMC) que mejor discriminan la sobrevida de la primera menstruación postparto. 3. Proponer un modelo predictivo que estime la probabilidad de la recuperación de la primera menstruación postparto al tiempo «১desde el tercer al doceavo mes postparto para un perfil de valores de las variables predictoras.

Definiciones usadas:

- Lactancia materna exclusiva: Lactancia materna como el único nutriente para los niños (sólo se agregaron gotas de vitaminas por normativa chilena).

La condición de lactancia materna exclusiva se supeditó a que el incremento de peso del niño fuera normal.

- Primera menstruación postparto: Cualquier sangrado que ocurra separado por 10 días o más del sangrado del puerperio, el cual consiste en un sangrado normal o de tres días consecutivos de goteo sanguíneo y seguido por un segundo sangrado dentro de los 60 días.

Toma de muestra para la medición hormonal: Se obtuvo dos muestras de sangre de la madre en tomo al tercer mes postparto (89 a 107 días postparto) para realizar la medición hormonal plasmática. La primera muestra se obtuvo a media tarde $(16: 00 \mathrm{~h})$, entre $90 \mathrm{y}$ 120 min después de un episodio de amamantamiento y la segunda muestra se obtuvo $30 \mathrm{~min}$ después de iniciado un nuevo episodio de succión.

Las muestras fueron centrifugadas y el plasma fue almacenado a $-20^{\circ} \mathrm{C}$ hasta su medición de PRL y $\mathrm{E}$ por radioinmunoensayo.

Prolactina basal: Concentración de prolactina plasmática (mUI/L) obtenida antes del episodio de succión. Prolactina postsucción: Concentración de 
prolactina plasmática (mUI/L), obtenida a los 30 min de iniciado el episodio de succión. Estradiol: Concentración de E plasmático (pmol/L) obtenido en la primera muestra de sangre. Medición hormonal: Las concentraciones de PRL y E fueron realizadas mediante radioinmunoensayo (RIA), usando reactivos y metodología del Programa Medición de Hormonas Reproductivas de la Organización Mundial de la Salud ${ }^{10}$.

Etnia: Se determinó de acuerdo al número de apellidos de origen mapuche (principal grupo étnico autóctono de la región). Se clasificó como de etnia mapuche a las madres que poseían ambos apellidos de origen mapuche y como no mapuche cuando tenían uno o ningún apellido de origen mapuche.

Análisis estadístico. El modelo estadístico usado es el modelo de azar proporcional de $\operatorname{Cox}^{12}$. Teniendo como variable respuesta el tiempo de aparición de la primera menstruación postparto y como variables predictoras los niveles hormonales al tercer mes postparto: PRLb, PRLp y E. Estas tres variables hormonales fueron dicotomizadas para evitar el problema de no linealidad y simplificar la interpretación de los resultados. Se incluye, además, entre las variables predictoras, los indicadores biodemográficos: edad, etnia, paridad e IMC materno. El supuesto de proporcionalidad de azar se revisa mediante el gráfico log-log.

Método para la selección del punto de corte en las variables hormonales: Se genera sistemáticamente los puntos de corte a lo largo del rango observado de cada variable, dicotomizándola, es decir generando dos grupos, los casos que estén bajo dicho punto de corte y los que estén sobre dicho valor. No se consideran los puntos de corte en los extremos de la variable, dado que, naturalmente, la frecuencia de casos en los extremos es muy baja y no permitina estimar la razón de azar con una precisión adecuada y en consideración al punto de vista clínico que indica que el punto de corte de una variable homonal, es decir el que mejor discrimine el tiempo de amenorrea de lactancia, debenía ser un valor que no se encuentre en los extremos de la variable. Teniendo como variable de respuesta, el tiempo de aparición de la primera menstruación postparto, a cada dicotomización de las variables homonales, se ajustó un modelo de azar proporcional, registrando el estimador puntual y por intervalo de confianza (IC) 95\% de la razón de azar comespondiente. El conjunto de puntos de corte y la respectiva estimación de la razón de azar, fue graficado, seleccionando el punto de corte, dentro de la tendencia, al que se asocia simultáneamente a la más alta razón de azar con un nivel de precisión en la estimación razonablemente buena» y significativa (que el IC no contenga el valor 1) ${ }^{13}$. Modelo estadístico: El modelo de Cox completo utilizado contiene como variables predictoras, la edad, paridad, IMC, etnia y las variables hormonales dicotomizadas. El modelo final selecciona aquellas variables que mejor predicen la aparición de la primera menstruación postparto al tiempo t.

El software estadístico usado es Stata 9.0.

\section{RESULTADOS}

Descripción del grupo en estudio. Se analizan 81 casos que cumplían los criterios de selección del estudio. La Tabla 1 muestra el perfil biodemográfico de las madres estudiadas.

Se registró 34,6\% de primíparas y 23,5\% con dos apellidos mapuches. La edad gestacional al parto varió entre las 37 y 42 semanas, con un promedio

Tabla 1. Promedio, desviación estándar valores mínimo y máximo de la edad, peso, talla e índice de masa corporal de las 81 madres del estudio

\begin{tabular}{|lrrrr|}
\hline Variable & Promedio & DSt. & Mín & Máx \\
\hline Edad (años) & 24,96 & 4,45 & 18 & 36 \\
Peso $(\mathrm{kg})$ & 59,62 & 8,41 & 44 & 80 \\
Talla $(\mathrm{cm})$ & 154,69 & 5,07 & 140 & 165 \\
IMC & 24,93 & 3,42 & 18,1 & 33,8 \\
\hline
\end{tabular}


de 39,85 semanas $\pm 1,25$ (DSt). El 92,5\% quedó con un método de anticoncepción no hormonal y el resto no usó método anticonceptivo.

La Tabla 2 muestra el promedio, desviación estándar y valor mínimo-máximo de las concentraciones de PRLb, PRLp y E a los 90 días postparto $\pm 3,00$ días (DSt).

Análisis de sobrevida. El tiempo mediano de sobrevida (tiempo donde la probabilidad de recuperar la menstruación es de 50\%) alcanzó los 209 días desde el parto. La Figura 1 resume el tiempo de sobrevida para los 81 casos.

Las Figuras 2-5, muestran la estimación de la razón de azar (el riesgo instantáneo de sufrir el evento por unidad de tiempo) para diferentes puntos de corte de las variables hormonales, además en éstas se señala el punto de corte escogido para el análisis. Estas figuras fueron usadas para determinar el punto de corte que mejor discrimina en la predicción la aparición de la menstruación. Cada una de las variables dicotomizadas mediante el punto de corte escogido, mostró respetar razonablemente el supuesto de proporcionalidad del azar.

Con los puntos de corte señalados en las cuatro figuras anteriores, se generaron las tres variables hormonales dicotomizadas. Se ajustó un modelo de Cox para la estimación de la sobrevida (aparición de la primera menstruación postparto) con todas las variables. En dicho modelo, sólo fueron significativas para la predicción, las variables hormonales dicotomizadas E (190 pmol/l) y PRLp (2,550 mUI/L).

El modelo estimado final es el siguiente:

$\mathrm{S}(\mathrm{t} / \mathrm{PLRp} 2550, \mathrm{E} 190)=\mathrm{S}_{\text {basal }}(\mathrm{t}) \exp (-0,5544651 \cdot \mathrm{PLRp} 2550+0,5064639 \cdot \mathrm{E} 190)$

Donde S(t/PLRp2.550, E190) representa la probabilidad de seguir amenorreica después del tiempo t $(90<t<500$ días), dado un perfil de PLRp

Tabla 2. Promedio, desviación estándar y valor mínimo y máximo para las tres variables hormonales

\begin{tabular}{|lcccc|}
\hline Variable hormonales & Promedio & DSt. & Mín & Máx \\
\hline Prolactina basal mUI/L & $1.492,5$ & 946,83 & 264 & 5.044 \\
Prolactina postsucción mUI/L & $2.614,4$ & $1.667,24$ & 553 & $1.031,3$ \\
Estradiol pmol/l & 183,7 & 99,33 & 40 & 622 \\
Razón prolactina postsucción/estradiol & 20,8 & 21,85 & 1,29 & 124,5 \\
\hline
\end{tabular}

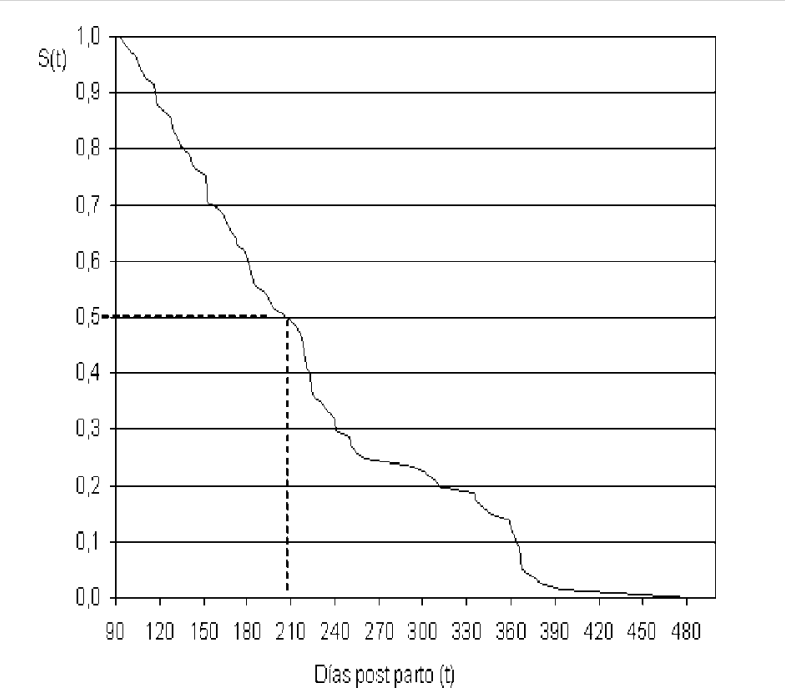

Figura 1. Probabilidad de permanecer amenorreica más allá de los 90 días postparto (al tiempo $t$, sobrevida $\mathrm{S}(\mathrm{t}))$ estimada por el método de Kaplan-Meier. 


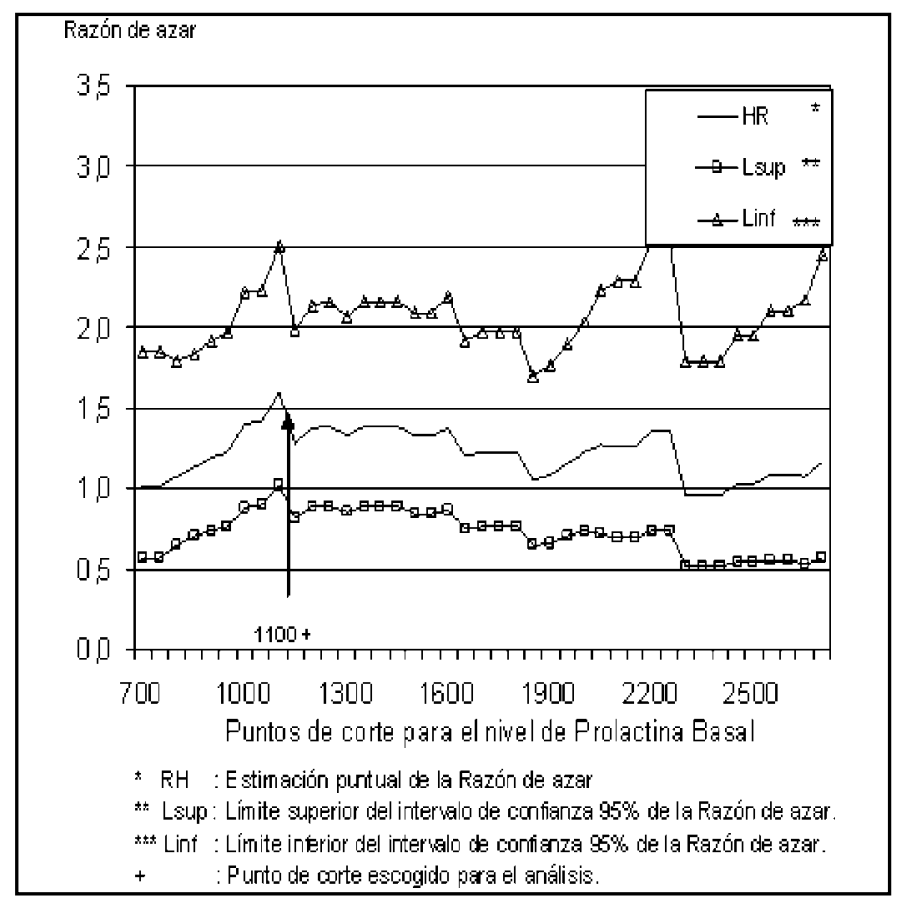

Figura 2. Estimación puntual y por intervalo de confianza 95\% para la razón de azar en distintos puntos de corte de prolactina basal.

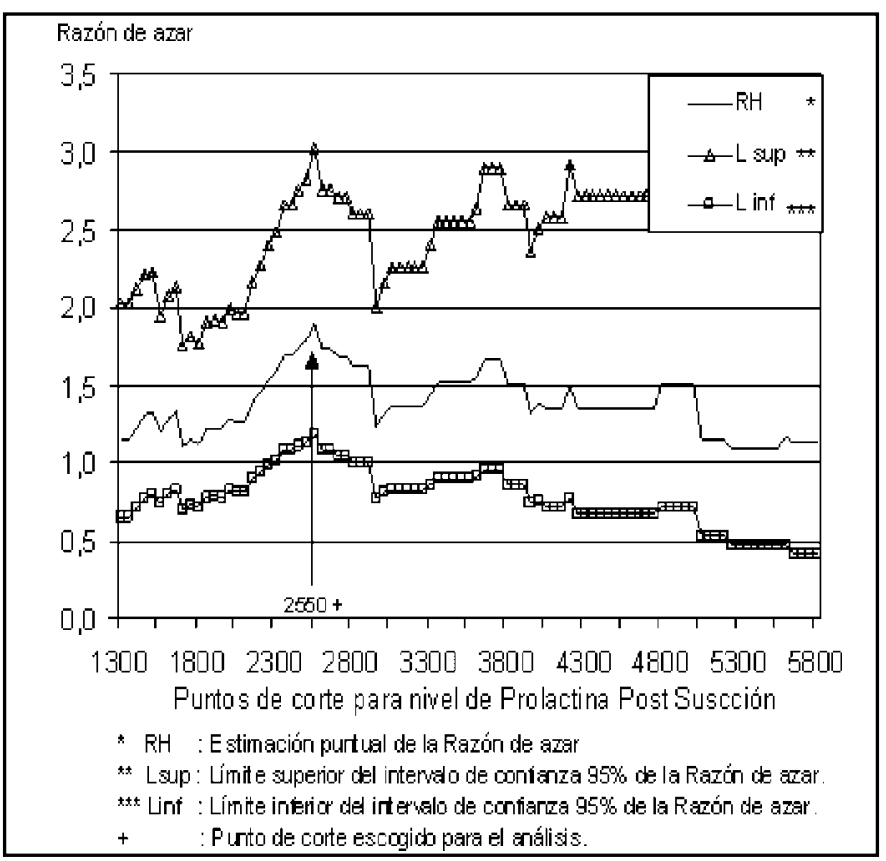

Figura 3. Estimación puntual y por intervalo de confianza 95\% para la razón de azar en distintos puntos de corte de prolactina postsucción.
(PLRp2550 $=0$ si PLRp es menor o igual a $2.550 \mathrm{mUI} / \mathrm{L}$ o 1 si PLRp es mayor que $2.550 \mathrm{mUI} / \mathrm{L})$ y E $(\mathrm{E} 190=0 \mathrm{si} \mathrm{E}$ es menor o igual a $190 \mathrm{pmol} / \mathrm{l}$ o $1 \mathrm{si} \mathrm{E}$ mayor que $190 \mathrm{pmol} / \mathrm{l}$ ); $\mathrm{S}_{\text {basal }}$ es la función de sobrevida basal para individuos con PRLp $\leq 2.550$ y $E \leq 190$.

Con el fin de observar independientemente el efecto en la sobrevida de las dos variables predictoras del modelo, se presentan en la Figura 6 para cada combinación de sus categorías, las curvas estimadas por el modelo de Cox. En esta figura, se observa que las mujeres que al tercer mes postparto presentan una medición de PLRp mayor a 2.550 y simultáneamente $\mathrm{E}$ igual o inferior a 190 tienen un riesgo de recuperar la menstruación menor que aquellas mujeres que tienen E sobre 190 o PLRp de 2.550 o menor; y mucho más bajo aún que aquellas mujeres que tengan simultáneamente E sobre 190 y PLRp de 2.550 o menos. A modo de ejemplo, la sobrevida mediana estimada por el modelo de Cox del grupo más protegido ( $E \leq 190$ y PLR $>2.550$ ) es de 240 días, en cambio en el grupo de mayor riesgo ( $\mathrm{E}>190$ y $\mathrm{PLR} \leq 2.550$ ) es de sólo 170 días. Al comparar la sobrevida de los cuatro grupos de riesgo mediante el test de Log-rank y ajustando por comparaciones múltiples mediante el método de Sidak, se observa que sólo se distingue significativamente (valor $p<0,05$ ) los grupos extremos, el más protegido ( $\mathrm{E} \leq 190 \mathrm{y}$ PLR >2.550) y el de mayor riesgo ( $\mathrm{E}$ $>190$ y PLR $\leq 2.550$ ). La Tabla 3 muestra la sobrevida observada para cada combinación de las variables de riesgo, incluyendo el tiempo total en riesgo (días), la tasa de incidencia (días persona), y los tres cuartiles de sobrevida. Adicionalmente, la Tabla 4 muestra los percentiles de sobrevida $5,10,25,50,75,90$ y 95 predichos por el modelo de Cox. 
Figura 4. Estimación puntual y por intervalo de confianza 95\% para la razón de azar en distintos puntos de corte de estradiol.
Figura 5. Estimación puntual y por intervalo de confianza 95\% para la razón de azar en distintos puntos de corte de la razón PRLp/E.
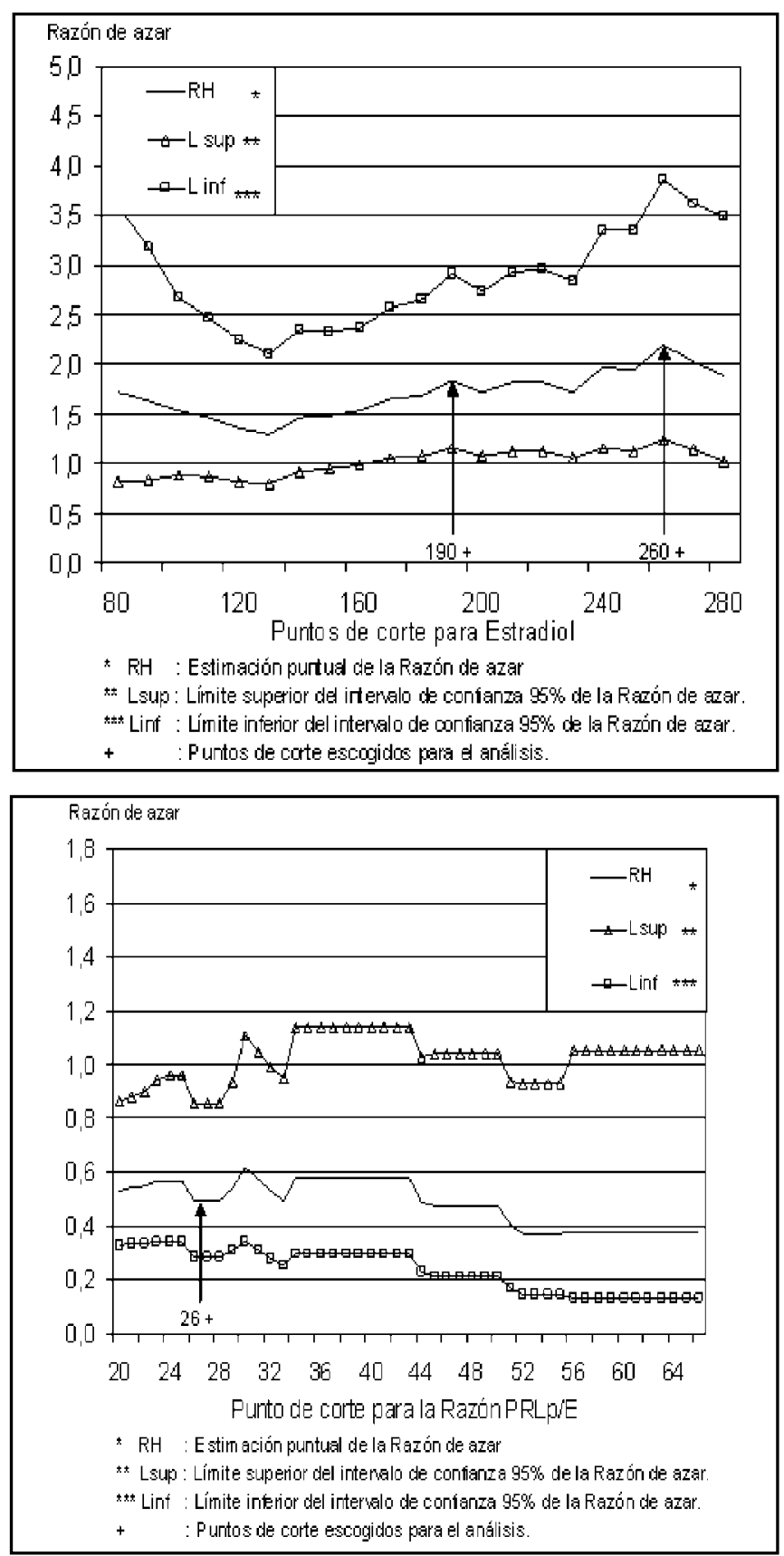

\section{Discusión}

La mayoría de los estudios sobre duración de la $\mathrm{AL}$, clasifican la duración de la AL como cortas o largas, de acuerdo a la respectiva presencia o no de menstruación al sexto mes postparto. Con esta definición se limitan a comparar los grupos de AL «cortas» y ঝargas» en función de las variables de exposición, tales como las variables hormonales ${ }^{14}$. Esta forma de análisis identifica las variables que distinguen los dos grupos de AL, pero no permite tener una idea con una adecuada precisión de 


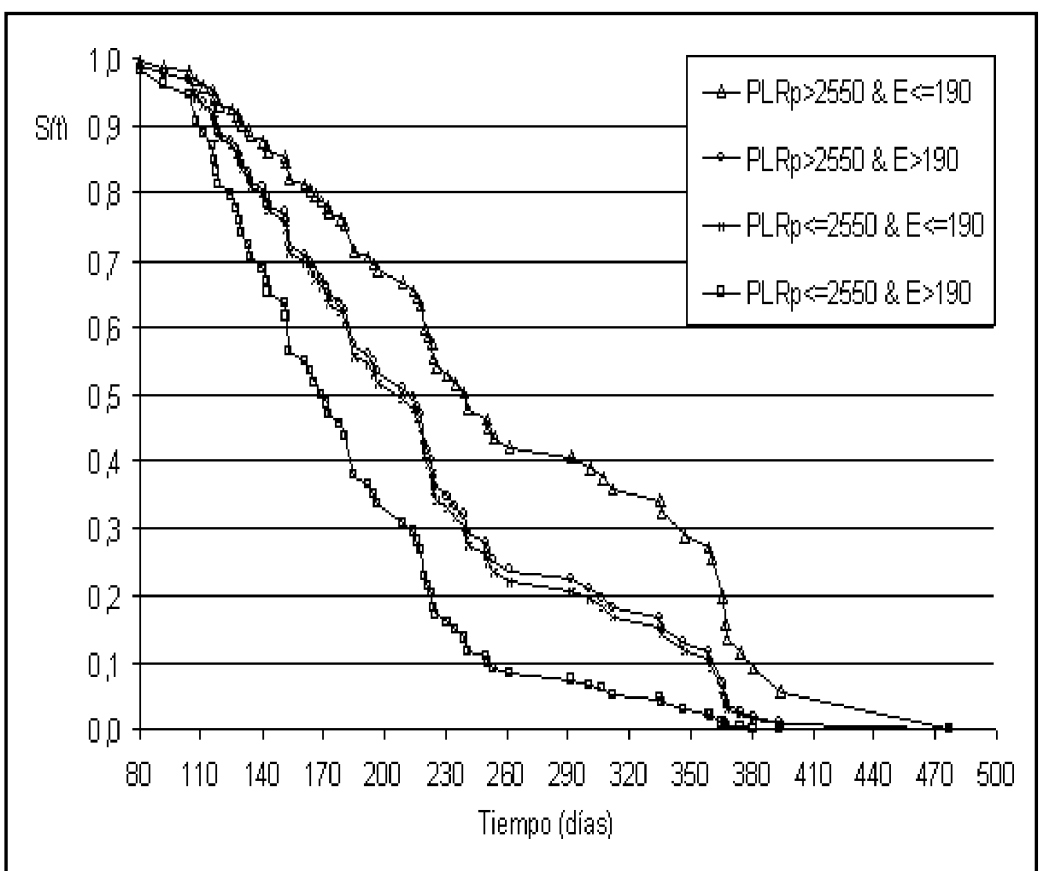

Figura 6. Curvas de sobrevida para todas las combinaciones de las variables predictoras. Sobrevida estimada por el modelo de Cox.

Tabla 3. Tasa de incidencia y cuartiles de sobrevida total y según grupos de riesgo observados en el estudio

\begin{tabular}{|lccrrrr|}
\hline Grupos de riesgo & $\begin{array}{c}\text { Tiempo } \\
\text { en riesgo }\end{array}$ & $\begin{array}{c}\text { Tasa de } \\
\text { incidencia }\end{array}$ & \multicolumn{2}{c|}{ № } & \multicolumn{3}{c|}{ Tiempo sobrevida } \\
personas & $25 \%$ & $50 \%$ & $75 \%$ \\
\hline E $\leq 190$ \& PLRp $\leq 2.550$ & 6.056 & 0,00462 & 28 & 153 & 224 & 254 \\
E $>190$ \& PLRp $\leq 2.550$ & 4.000 & 0,00575 & 23 & 128 & 164 & 209 \\
E $\leq 190$ \& PLRp $>2.550$ & 6.099 & 0,00361 & 22 & 185 & 312 & 366 \\
E $>190$ \& PLRp $>2.550$ & 1.741 & 0,00460 & 8 & 135 & 220 & 301 \\
Total & 17.896 & 0,00453 & 81 & 152 & 209 & 261 \\
\hline
\end{tabular}

Tabla 4. Tasa de incidencia y cuartiles de sobrevida total y según grupos de riesgo observados en el estudio

\begin{tabular}{|lrrrrrrr|}
\hline & \multicolumn{7}{c|}{ Percentiles de sobrevida } \\
Grupos de riesgo & \multicolumn{1}{c}{5} & 10 & 25 & 50 & 75 & 90 & 95 \\
\hline E $\leq 190$ \& PLRp $\leq 2.550$ & 105,4 & 117,5 & 151,3 & 201,7 & 250,2 & 359,7 & 366,5 \\
E $>190$ \& PLRp $\leq 2.550$ & 96,6 & 110,2 & 129,6 & 166,1 & 219,2 & 250,2 & 331,5 \\
E $\leq 190$ \& PLRp $>2.550$ & 116,7 & 132,8 & 184,0 & 240,8 & 365,7 & 377,7 & 468,1 \\
E $>190$ \& PLRp $>2.550$ & 105,0 & 117,1 & 152,8 & 211,7 & 260,9 & 365,6 & 366,2 \\
\hline
\end{tabular}


cuál es la probabilidad de que una mujer recupere la fertilidad a un mes determinado dentro del primer año postparto.

El modelo de análisis que propone este estudio, utilizando el modelo de Cox, permite estimar el riesgo de recuperar la menstruación o de permanecer amenorreica más allá de un tiempo definido entre los tres y los doce meses postparto, de acuerdo a los niveles de PRLp y E medido al tercer mes postparto. La innovación de este modelo es que no compara transversalmente dos grupos de mujeres (amenorreicas y no amenorreicas al completar el sexto mes postparto), sino que evalúa el tiempo de sobrevida (tiempo a la aparición de la primera menstruación post parto) para un perfil determinado de madres en lactancia exclusiva. La potencial aplicación de este modelo a nivel clínico debería ser de tipo computacional, donde se ingrese el perfil del caso a un tiempo de predicción definido por el clínico, mediante lo

\section{REFERENCIAS}

1. Short RV. Breast-Feeding. Sci Am 1984; 250: 3541.

2. Mc Neiшy AS. Effects of lactation on fertility. $\mathrm{Br}$ Med Bull 1979; 35: 151-54.

3. Wood JW, Johnson P L, CAmpbel KL. Demographic and endocrinological aspects of low natural fertility in highland New Guinea. J Biosoc Sci 1985; 17: 57-9.

4. Díaz S, Rodríguez G, Casado ME, Miranda P, SchiappaCASSE V, BRANDEIS A ET al. Breastfeeding pattern and the duration of lactational amenorrhea, ovulation and infertility in urban Chilean women. Contraception 1988; 38: 37-51.

5. Glasier A, McNeiLy AS, Howie PW. The prolactin response to suckling. Clin Endocrinol 1984; 21: 109-16.

6. Díaz S, Seron-Ferre M, Cárdenas H, Schiappacasse V, Salvatierra AM, Herreros C et al. Early Difference in the Endocrine Profile of Long and Short Lactational Amenorrhea. J Clin Endocrinol Metab 1991; 72: 196-01.

7. Díaz S, Seron-Ferre $M$, Cárdenas $H$, Schiappacasse V, Brandeis A, Croxatto HB. Circadian Variation of Basal Plasma Prolactin, Prolactin Response to Suckling and Length of Amenorrhea in Nursing cual el software estimaría la probabilidad de recuperación de la primera menstruación postparto a dicho tiempo (version preliminar en www.ciges.cl/docentes/jjorell/al.xls).

Finalmente, es necesario señalar que el modelo propuesto debe ser sometido a validación en un grupo independiente de casos, que es posible y necesario mejorar la precisión de la estimación del riesgo, incluyendo otras variables predictoras como características del amamantamiento. Por otro lado, se debe evaluar si existen variaciones clínicamente significativas en las concentraciones hormonales de PRLp y E después del tercer mes postparto entre mujeres con amenorreas cortas y largas y que mantengan lactancia exclusiva hasta el sexto mes postparto. En caso de registrarse variaciones significativas, las variables de concentraciones hormonales deben ser incorporadas dentro del modelo como variables dependientes del tiempo.

Women. J Clin Endocrinol Metab 1989; 68: 946-55.

8. Campino C, Ampuero S, Díaz S, Seron-Ferre M. Prolactin bioactivity and the duration of lactational amenorrhoea. J Clin Metab 1994; 79: 970-4.

9. Campino C, Ampuero S, López JM, Serón-Merre S. Postsuckling Prolactin: Oestradiol ratio-a potential index to predict the duration of Lactational Amenomhoea in women. Human Reproduction 1997; 12: 1421-6.

10. WHO Special Programme of Research Development and Research Training in Human Reproduction. Programme for the Provision of watched. Assay Reagents for the RIA of Hormones in Reproductive Physiology Method Manual, 11 th Ed. 1987.

11. Valdés P, Sierralta P, Ossa X, BarRía A. Niveles de estradiol y duración de la amenorrea de lactancia. Rev Chil Obstet Ginecol 1993; 58: 211-5.

12. Kiein J, Moeschberger M. Statistics for Biology and Health. Survival Analysis. Techniques for Censored and Truncated Data. Spring-Verlag editors, New York, 1998.

13. Altman DG, Lausen B, Sauerbrei W, Schumacher M. Dangers of using «optimal» cutpoints in the evaluation of prognostic factors. J Natl Cancer Inst 1994; 86: 829-35.

14. Vaidés P, Sierralta P, Ossa X, Barría A. Duración de la amenorrea de lactancia en una población urbana de Temuco. Rev Méd Chile 2002; 130: 66-70. 